\title{
Design Thinking to Support the Development of a Safe Web- Based Health Information Seeking Platform for Older Adult Populations
}

This paper was downloaded from TechRxiv (https://www.techrxiv.org).

\section{LICENSE}

CC BY 4.0

SUBMISSION DATE / POSTED DATE

24-09-2021 / 29-09-2021

\section{CITATION}

Shaveet, Eden; Gallegos, Marissa; Castle, Jonathan; Bryant, Alison; Gualtieri, Lisa (2021): Design Thinking to Support the Development of a Safe Web-Based Health Information Seeking Platform for Older Adult Populations. TechRxiv. Preprint. https://doi.org/10.36227/techrxiv.16679776.v1

$\mathrm{DOI}$ 


\section{Design Thinking to Support the Development of a Safe Web-Based Health Information Seeking Platform for Older Adult Populations}

Eden Shaveet, BA, CPS

Tufts University School of Medicine

Marissa Gallegos, BS

Tufts University School of Medicine

Jonathan Castle, BFA

Tufts University School of Medicine

Alison Bryant, PhD, MA

AARP

Lisa Gualtieri, PhD, ScM

Tufts University School of Medicine

Abstract- The pervasiveness of online mis/disinformation escalated during the COVID-19 pandemic. To address the proliferation of online mis/disinformation, it is critical to build safety into the tools older adults use to seek health information. On average, older adult populations demonstrate disproportionate susceptibility to false messages under the guise of informative authority and were the most engaged with false information about COVID-19 across online platforms when compared to other age-groups. In a design-thinking challenge posed by AARP to graduate students in a Digital Health course at Tufts University School of Medicine, students leveraged existing solutions to develop a health information platform that is responsive to both passive and active health information-seeking methods utilized by older adults in the United States. This paper details the design-thinking process employed, results of primary research, an overview of the prototyped platform, and insights relating to the design of effective health information-seeking platforms for older adults.

Adults $\mathbf{5 0}$ years and older are the largest consumer population of healthcare in the United States [1]. Consequently, the ways older adults seek health information is noteworthy for healthcare providers, older adult communities, and public health stakeholders. The number of older adults seeking health information online has increased substantially in recent years [2]; however, increased reliance on online health information seeking has heightened concern around rapid dispersion of misinformation and disinformation in the online health space [3].

ONLINE HEALTH INFORMATION SEEKING DURING COVID-19

Disruption of routine healthcare service delivery in the U.S. resulting from the COVID-19 pandemic prompted increased patient-reliance on internetenabled technologies for virtual visits with providers, consumer-grade digital health application use, and vaccination appointment scheduling [4]. This

Digital Object Identifier XXXXXXXX 
heightened dependence contributed to a greater overall reliance on online health information-seeking behaviors. In 2020 , over $80 \%$ of U.S. adults aged 50 years or older claimed that the internet had been an essential resource to them throughout the pandemic [5]; however, older adults were also found to be the most engaged with false information about COVID-19 across online platforms when compared to other agegroups [6].

\section{MISINFORMATION AND DISINFORMATION}

Misinformation and disinformation refer to false messaging spread in a variety of contexts under the guise of informative content [7]. While misinformation is defined simply as false information, disinformation is the sect of misinformation that is deliberately propagated [7]. In recognition of their mutual relevance and harm, we will use the two terms collectively throughout this paper. Concern surrounding the proliferation of online mis/disinformation has garnered considerable public attention in recent years [7]. Despite its prevalence on online platforms with youthskewed user-bases, older adult populations are, on average, disproportionately susceptible to mis/disinformation when encountered [3].

While its presence has drastically increased since the early 2010's, pervasiveness of online health mis/disinformation is broadly believed to have escalated during the COVID-19 pandemic. In the online health space, one may assume optimal solutions to mitigating proliferation include both reducing the amount of false content in online circulation and promoting better health and digital literacy skills. However, these are massive endeavors requiring substantial time and resources. Proposed in this paper is a solution with fewer barriers to implementation.

\section{TARGET POPULATION}

Older adults who seek health information online are often prompted by receipt of new diagnoses, progression of symptoms, or prescription of new medications [8]. Given that $85 \%$ of adults 50 years and older live with at least one chronic condition that may change over time or require medication [9], we identified this population as the target user base for a design-thinking solution.

\section{DESIGN THINKING}

Design thinking, an approach to innovation and validation, is utilized to develop effective solutions to complex problems [10]. Distinct from typical web platform design processes, design thinking leverages human-centered design principles to observe how people interact with their environments and design to a user population's expressed needs. Student teams at Tufts University School of Medicine engaged in a design-thinking process adapted to a 14-week Digital Health course in collaboration with AARP (a U.S. member-based interest and advocacy group focusing on issues affecting adults over the age of 50) to address a set of obstacles facing older adults in the U.S. We engaged in a design-thinking methodology encompassing review of the literature and existing solutions, primary research, problem explication, ideation, prototyping, and solution validation to address the problem of unreliable health information seeking within older adult communities.

\section{WHY AARP?}

Trust is foundational to solution adoption and behavior change in older adult communities [11]. Given AARP's tenure in the older adult interest and advocacy space, the organization is one of the most well-known and respected membership-based organizations in the U.S. At a membership count of over 38 million people, AARP is a household name frequently referenced in pop-culture, academic, and industry spaces.

\section{AARP AND INFORMATION}

In their efforts to mitigate online mis/disinformation about COVID-19, Google's early decision to promote COVID-19 information sourced from AARP amplified the organization's credibility as a trusted source for health information [12]. For this reason, along with the substantial use of their website by older adults, AARP was an ideal entity with which to prototype a highimpact design-thinking solution to the problem of unreliable health information seeking online.

\section{ASSESSMENT OF PRESENT SOLUTIONS}

The internet is a primary source of health information gaining popularity within older adult communities [1]. Online health information seekers tend to use Google search for health-related queries [1]. Since health literacy skills are poor among many health information seekers [2], safe and trusted searches may be achieved through 1.) training health information seekers to identify valid content (a massive undertaking) or 2.) employing methods that in some 
way limit search or search results, such as using specialized search engines or seeking content which meet verification criteria.

\section{Specialized Search Engines}

Several specialized health search engines have emerged in the last two decades; however, historically, these search engines have been unsuccessful in reaching wide-audiences when compared to Google or mitigating the proliferation of health-related mis/disinformation online [13].

\section{Content Certification Programs}

Online health content certification programs inform users of access to websites housing validated health information [14]. However, low adoption of these programs implies infrequent recognition of their certification indicators across the general population. The success of content certification programming depends on online health information seekers recognizing and valuing these indicators.

\section{AARP-BASED SOLUTIONS}

\section{AARP Health Tools}

AARP hosts a suite of 12 web-based health tools for their members on their website including a pill identifier, symptom checker, and health encyclopedia populated by content licensed from Healthline's Health Reference Library [15]. While valuable, this content is not intuitively located nor easy to access on the AARP website.

\section{AARP Perks ${ }^{\mathrm{TM}}$ Browser Extension}

AARP's Perks ${ }^{\mathrm{TM}}$ browser extension notifies members of AARP benefits while browsing online [16]. Though not designed for the purpose of health information-seeking, the extension was designed based on the online information-seeking habits of older adults, including passive information acquisition while browsing for other information.

\section{Methods}

The design thinking process included user research, platform research, validation, and solution ideation. We report on the processes selected and used in our Methods section.

\section{USER RESEARCH}

The design thinking process emphasizes understanding the needs and constraints of the target population. To accomplish this, we conducted key informant interviews.

\section{$\underline{\text { Key Informant Interviews }}$}

We convened a non-probability sample of key informants $(n=4)$ consisting of three target population members and one caregiver (See Table 1). Informants were asked about their health information seeking habits and experience with AARP.

\section{Table 1. Key Informant Characteristics}

\begin{tabular}{lll} 
Age (years) & $\boldsymbol{n}$ & $\%$ \\
\hline $40-45$ & 1 & 25 \\
$70-75$ & 1 & 25 \\
$80-85$ & 1 & 25 \\
$85-90$ & 1 & 25 \\
\hline Sex & $\boldsymbol{n}$ & $\%$ \\
\hline Male & 3 & 75 \\
Female & 1 & 25 \\
\hline Race & $\boldsymbol{n}$ & $\%$ \\
\hline White & 2 & 50 \\
Inuit indigenous & 2 & 50 \\
\hline Highest education completed & $\boldsymbol{n}$ & $\%$ \\
\hline Some college & 1 & 25 \\
Undergraduate degree & 1 & 25 \\
Graduate degree & 2 & 50 \\
\hline Annual income (USD) & $\boldsymbol{n}$ & $\%$ \\
\hline Under 50k & 1 & 25 \\
60-70k & 2 & 50 \\
>100k & 1 & 25 \\
\hline
\end{tabular}

\section{PLATFORM RESEARCH}

AARP's existing partnerships for hosting validated health information online and their existing browser extension architecture informed our primary research process. We evaluated AARP platform features including the main search bar and Perks browser extension functionality to observe their underlying mechanisms, identify barriers and facilitators of health information access, and identify opportunities to leverage these features in a feasible health information seeking solution. User-experience research included assessing AARP's health content click path, scroll depth, and readability.

\section{Search Bar Evaluation}

We conducted an evaluation of AARP's website search bar to observe content recall and page rank. Over the course of seven days, we utilized three cookie and cache cleared web browsers to submit 126 queries of six distinct health-related terms in the following categories: medical conditions, medications, and health products (see supplemental materials). Forty-two queries on AARP.org were conducted on a locationtracking disabled web browser (DuckDuckGo) and 84 
queries were conducted on two location-tracking enabled web browsers (Microsoft Edge, Google Chrome). Different browsers with different tracking permissions were utilized to observe whether health content recall and page rank would be affected by the presence or lack of permissions.

Browser Extension Evaluation

We conducted an evaluation of the AARP Perks ${ }^{\mathrm{TM}}$ web browser extension to infer the mechanics of the extension's search function and search-activated notification tab. We tested responsiveness to health and non-health related search terms and quantified instances of tab presence across search contexts to surmise algorithm structure (see supplemental materials).

Click Path and Scroll Depth Analysis

Click path and scroll depth are metrics which describe a user's experience on a website or platform [17]. Click path characterizes the number of clicks needed to access information of interest from a defined start point (often the website homepage), while scroll depth characterizes the amount of scrolling necessary to access content between clicks. In the interest of minimizing transaction costs which dissuade user retention, minimal click path and scroll depth may be desirable. Our user experience analyses assessed the shortest possible click path and scroll depth required to access clinically validated health information hosted on the AARP website.

Content Readability Comparison Test

As information access also depends on its readability, online content hosted by AARP pertaining to the COVID-19 vaccination was assessed and compared to online anti-vaccination content via six readability indices: Flesch Kincaid Reading Ease, Flesch Kincaid Grade Level, Gunning Fog Score, SMOG Index, Coleman Liau Index, and the Automated Readability Index.

\section{VALIDATION AND SOLUTION IDEATION}

We sought validation of research findings with AARP's digital analytics architect and senior advisor of enterprise search. Greater insight was provided into the behavior of AARP's existing solutions which corroborated with our intentions to develop a solution that would be implementable with the existing architecture.

\section{Results}

The Results section reports on findings distilled from the processes outlined above.

\section{KEY INFORMANT INTERVIEWS}

Key informants largely preferred seeking health information offline for queries about their own health or the health of others (50-75\%). Informants expressed low trust in commercial search engines and that privacy while browsing for health information online is of high importance to them $(75 \%)$. However, despite mistrust in commercial search engines, most informants expressed interest in using online health-information solutions, such as health search engines $(75 \%)$. Half of the informants were AARP members, but $0 \%$ had ever visited the AARP website and had thus never utilized AARP Health Tools. Additionally, no respondents were familiar with the Perks Browser Extension. These findings suggested a need for health information solutions to preserve privacy and be accessible in both online and offline formats. See supplemental materials.

\section{SEARCH BAR EVALUATION}

Page rank remained identical for all queries across testers and browsers. Notably, information sourced from AARP Health Tools (Health Encyclopedia, Symptom Checker, etc.) was not included in content recall. These findings led us to suspect use of an objectoriented mechanism for content recall, but no use of location-based relevance algorithms to modify page rank. We also suspected use of a self-contained content repository that excludes validated health content hosted by AARP for Health Tools.

\section{BROWSER EXTENSION EVALUATION}

\section{Extension Search Bar}

Six health and six non-health related terms were submitted to the Perks web browser extension search bar. Health-related search terms returned zero results, while non-health-related terms returned 15-30 results. Multi-word non-health related search terms returned 02 results. These findings led us to suspect use of lexical parsing not conducive to diversions from single-word query submissions resembling natural language.

\section{Extension Notification Tab}

The Perks extension notification tab did not appear upon submission of search terms into partner web browsers and search engines alone. It was not until accessing certain first page ranked results that the tab appeared with content relating to the search term. Validated health information sourced from AARP 
Health Tools was not present in content provided in the notification tab for submission of health-related terms, though a link to access the "symptom checker" health tool was sometimes returned. These findings led us to suspect use of a whitelisting algorithm to prompt appearance of the tab upon access of whitelisted universal resource locators (URLs), but it remained unclear as to why the symptom checker tool was returned on only certain whitelisted health-condition related URLs.

CLICK PATH AND SCROLL DEPTH ANALYSIS

The shortest possible path to validated health information on AARP.org through Health Tools contained a total of nine steps: seven clicks and two scroll events comprising $75 \%$ total scroll depth (See supplemental materials). Notably, this path required that users know and submit exact search terms. These findings led us to suspect that most website users are unsuccessful in locating this content passively or actively. This conclusion was consistent with the lack of key informants who had ever accessed Health Tools content.

\section{CONTENT READABILITY COMPARISON TEST}

When compared to online anti-vaccination content [18], a select AARP article pertaining to COVID-19 and vaccination options [19] ranked less readable in five out of six readability indices: Flesch Kincaid Reading Ease (11.8 pts compared to 55.2 pts), Flesch Kincaid Grade Level (13.9 pts compared to 8.6 pts), SMOG Index (9.2 pts compared to $8.4 \mathrm{pts}$ ), Coleman Liau Index (22 pts compared to 13.3 pts), and Automated Readability Index (13.5 pts compared to 7.9 pts). AARP content ranked more readable in only one readability index: Gunning Fog Score (8.9 pts compared to $9.1 \mathrm{pts}$ ). See supplemental materials for formulas. As content readability is central to access, these findings suggest that validated health content about COVID-19 hosted by AARP may be less accessible than certain online anti-vaccination mis/disinformation.

\section{Discussion}

The Discussion section outlines the design-thinking solution culminating from the insights detailed in our Results section. Outlined function of existing AARP products supported our belief that a high impact designthinking solution could leverage existing AARP platform architecture and introduce features that make the content more accessible. Additionally, the solution would need to integrate expressed needs of older adult health information seekers, including privacypreservation and multi-platform availability.

Refinement of existing solutions may lead to highly feasible implementation and replication at hand-off as opposed to design and development of costly novel solutions.

\section{AARP HEALTH TOOLS 2.0}

AARP Health Tools 2.0 is a multi-spectral health information-seeking platform prototype designed to be responsive to both passive and active health information-seeking methods utilized by older adults in the United States.

Health Tools Browser Extension

Leveraging the existing Perks web browser extension architecture, Health Tools 2.0 would employ a similar whitelisting algorithm to notify users of trusted health information sources while browsing online. Modifications to existing architecture would include redirection of the content repository path to the Health Tools repository, and a rewriting of whitelisting conditions. Health Tools 2.0 extension whitelisting conditions include return of a "checkbox" notification upon validation of whitelisted URL access and return of a "null" notification upon access of non-whitelisted URLs coupled with a notification tab populated by Health Tools content (See Figure 1).

By rewriting the whitelisting conditions, the extension function changes from member benefits notifications to content validation and supply. See supplemental materials for models and sample user interfaces. Departing from the Perks extension, in the interest of privacy-preservation, the Health Tools 2.0 extension would not require member sign-in prior to installation or use. 


\section{Perks Extension Whitelisting}

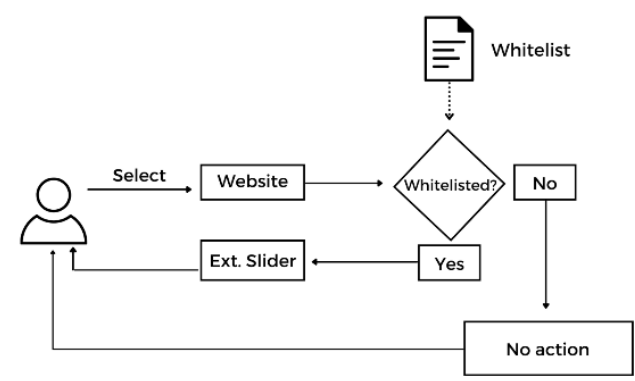

Health Tools (HT) Extension Whitelisting

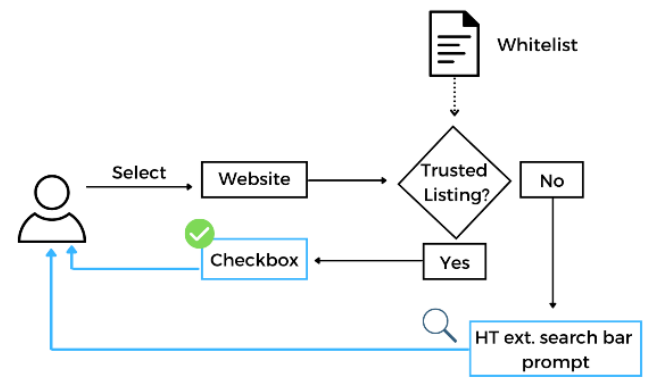

Figure 1. Notice the similarities between the AARP Perks $^{\mathrm{TM}}$ extension whitelisting algorithm in use (top) and the proposed Health Tools 2.0 extension algorithm (bottom). By rewriting whitelisting conditions, the extension function changes from member benefits notifications to content validation and supply.

\section{Webpage}

To address impediments to locating validated health content hosted by AARP, the Health Tools 2.0 prototype complements the browser extension with an easily navigable webpage solely dedicated to the 12 web-based health tools offered by AARP. Features of the page include embedded link buttons for each tool at $0 \%$ scroll depth, a large-sized search bar whose content repository is populated by content licensed from Healthline's Health Reference Library, and an introductory video to demonstrate use of Health Tools 2.0 (see supplemental materials).

Health Tools Augmented Search

Search functionality, a principal asset of Health Tools 2.0, is present in both a self-contained Health Tools search bar on the webpage and extension, as well as in a synchronic query parser to the main AARP website search bar. Queries submitted to the Health Tools search bar travel to the query parser where the indexed health content is recalled via an object-oriented mechanism. The content is ranked and returned to the user via the Health Tools user interface (website or extension tab). Additionally, queries submitted to the main AARP website search bar enter both the present query parsing and recall cycle employed by AARP as well as the Health Tools query parser where the indexed health content is recalled and returned to the user via a secondary content box that appears upon submission. See supplemental materials for models and sample user interfaces.

\section{ACCESSIBILITY}

To maximize the impact of Health Tools 2.0, focus on content accessibility is vital. We propose a dual approach to accessibility to support the efficacy of Health Tools 2.0:

Readability

Findings of the readability comparison tests revealed that published COVID-19 vaccination content on the AARP website [18] ranked less readable in five out of six readability indices when compared to online anti-vaccination content [19]. We propose the preliminary phase to Health Tools 2.0 adoption include review and revision of existing Health Tools content to the sixth grade reading level to broaden access.

\section{Cross-Platform Access}

Given the variability of information seeking techniques utilized by older adults in the US, information seeking platforms that aim to engage older adult audiences must be available across multiple mediums. Health Tools 2.0 can be adapted to multiple platforms by leveraging existing Perks web browser partnerships for extension adoption and offering Health Tools video series on AARP's video streaming channels. Additionally, to appeal to older adults who prefer to consume information offline, or for those not yet comfortable using digital technologies, Health Tools information can be made available in print media through AARP's print publications. Quick response (QR) codes in print media with step-by-step instructions may also provide an opportunity to support older adults in building digital literacy skills and encouraging reader access to safe health information online. 


\section{Conclusion}

Web-based health information seeking is on the rise across older adult populations in the U.S. and beyond. [1] In the absence of health information seeking solutions that accommodate the health informationseeking habits of older adults, the potential for exposure to health mis/disinformation escalates. The COVID-19 pandemic provides a case study of an infodemic in which older adults, the population most disproportionately susceptible to COVID-19, were also the most engaged with mis/disinformation that encouraged actions which enabled its spread [6] [20]. Health Tools 2.0 offers a framework for multi-platform health-information seeking solutions that may mitigate the factors that contribute to health misinformation cycles in older adult communities.

\section{KEY FINDINGS AND RECOMMENDATIONS}

Key findings of this work include that when responding to the problem of mis/disinformation proliferation in older adult communities, building upon existing tools may render a more feasible and impactful solution than a novel development. When modified, existing solutions targeting search habits of older adults may be used to mitigate access to health mis/disinformation online.

To promote adoption, these solutions could be operated or sponsored by entities older adults already trust. AARP is a trusted entity offering validated health information solutions and products which appeal to the online browsing habits of older adults; however, these products are disparate and may not be broadly accessible or delivered at the appropriate reading level. By consolidating these tools and making slight modifications to their function, a new solution emerges with a proposed ability to mitigate access to mis/disinformation online.

\section{Acknowledgment}

The authors express gratitude to Ted Moffatt, AARP Digital Analytics Architect, and Raymond Deschenes, AARP Senior Advisor of Enterprise Search, for their guidance and consultation following our research and preliminary ideation phase.

\section{Conflicts of Interest}

Eden, Marissa, Jonathan, and Lisa declare no conflicts of interest. Alison leads the Research Center at AARP but did not conduct the analyses detailed in this paper.

\section{- REFERENCES}

1. A. M. Turner, K. P. Osterhage, J. O. Taylor, A. L. Hartzler, and G. Demiris, "A Closer Look at Health Information Seeking by Older Adults and Involved Family and Friends: Design Considerations for Health Information Technologies," AMIA Annu. Symp. Proc. AMIA Symp., vol. 2018, pp. 10361045, 2018.

2 R. W. Berkowsky and S. J. Czaja, "Challenges associated with online health information seeking among older adults," in Aging, Technology and Health, Elsevier, 2018, pp. 31-48. doi: 10.1016/B978-0-12-811272-4.00002-6.

3. B. Swire-Thompson and D. Lazer, "Public Health and Online Misinformation: Challenges and Recommendations," Аnnи. Rev. Public Health, vol. 41, no. 1, pp. 433-451, Apr. 2020, doi: 10.1146/annurev-publhealth-040119-094127.

4 R. De', N. Pandey, and A. Pal, "Impact of digital surge during Covid-19 pandemic: A viewpoint on research and practice," Int. J. Inf. Manag., vol. 55, p. 102171, Dec. 2020, doi: 10.1016/j.ijinfomgt.2020.102171.

5. E. Vogels, A. Perrin, L. Rainie, and M. Anderson, " $53 \%$ of Americans Say the Internet Has Been Essential During the COVID-19 Outbreak," Pew Research Center, Apr. 2020. [Online].Available:

https://www.pewresearch.org/internet/2020/04/30/53-ofamericans-say-the-internet-has-been-essential-during-thecovid-19-outbreak/

6. OMA, "Report on COVID Research in Ontario for the OMA," Ontario Medical Association, Toronto, Canada, Mar. 2021. [Online].Available:

https:/www.oma.org/uploadedfiles/oma/media/public/covidmyths-asi-research-summary-for-oma.pdf

7. "Misinformation, Disinformation, and Online Propaganda," in Social media and democracy: the state of the field and prospects for reform, Cambridge New York Port Melbourne New Delhi Singapore: Cambridge University Press, 2020, pp. 10-27.

8. A. Ghazanfari and J. Chin, "What Triggers Health Information Search: An Explorative Analysis on the Information Needs, Situations, Technologies, and Habits in Health Information Search Across the Lifespan," Proc. Int. Symp. Hum. Factors Ergon. Health Care, vol. 8, no. 1, pp. 125-125, Sep. 2019, doi: 10.1177/2327857919081028.

9. NIH, "Supporting Older Patients with Chronic Conditions," National Institute on Aging, May 17, 2017. https://www.nia.nih.gov/health/supporting-older-patientschronic-conditions.

10. R. Linke, "Design Thinking, Explained,” MIT, Sep. 14, 2017. http://mitsloan.mit.edu/ideas-made-to-matter/designthinking-explained.

11. J. McMurray et al., "The Importance of Trust in the Adoption and Use of Intelligent Assistive Technology by Older Adults to Support Aging in Place: Scoping Review Protocol," JMIR Res. Protoc., vol. 6, no. 11, p. e218, Nov. 2017, doi: 10.2196/resprot.8772.

12. E. Moffatt and R. Deschenes, "Expert consult interview," Jun. 28, 2021.

13. R. Lyengar, "Why it's so hard to dethrone Google," CNN, Oct.22,2020.[Online].Available: https://www.cnn.com/2020/10/21/tech/google-antitrustlawsuit-better-search/index.html.

14. "HONcode Certification," Health on the Net. https://www.hon.ch/en/certification.html\#certification.

15. AARP, "Health Tools," AARP Health Tools. https://www.aarp.org/health/health_tools/. 
16. "AARP PerksTM Browser Extension," AARP, 2021. https://www.aarp.org/benefits-discounts/perks/install/.

17. T. Sinha, P. Jermann, N. Li, and P. Dillenbourg, "Your click decides your fate: Inferring Information Processing and Attrition Behavior from MOOC Video Clickstream Interactions," arXiv, Sep. 2014, Accessed: Sep. 17, 2021. [Online]. Available: http://arxiv.org/abs/1407.7131

18. S. Tenpenny, "Are Vaccines Necessary?," Vaccine Injury. https://vaccine-injury.info/tenpenny.cfm.

19. R. Nania, "What You Need to Know About the Coronavirus," AARP.https://www.aarp.org/health/conditionstreatments/info-2020/coronavirus-facts.html.

20. A. L. Mueller, M. S. McNamara, and D. A. Sinclair, "Why does COVID-19 disproportionately affect older people?," Aging, vol. 12, no. 10, pp. 9959-9981, May 2020, doi: 10.18632/aging. 103344 .

Eden Shaveet is a graduate student of health informatics and analytics at Tufts University School of Medicine, Boston, MA, USA. She received a B.A. in Psychology from Elms College in 2019. Eden currently pursues research and evaluation at UMass Chan Medical School. Contact her at eden.shaveet@tufts.edu.

Marissa Gallegos is a graduate student of health informatics and analytics at Tufts University School of Medicine, Boston, MA, USA. She received a B.S. In Biochemistry and Molecular Biology from Oregon State University in 2019. Marissa currently trains as a doula with the Community Doula Program in Oregon. Contact her at marissa.gallegos@tufts.edu.

Jonathan Castle is a graduate student of health informatics and analytics at Tufts University School of Medicine, Boston, MA, USA. He received a B.F.A in Visual Communications from American Intercontinental University in 2009. After serving twenty years as an U.S. Army medic, Jonathan pursues health equity and service to underserved communities as a data analyst at Elevate Health. Contact him at jonathan.castle@tufts.edu.

Alison Bryant, PhD, MA is the Senior Vice President of Research \& Enterprise Lead for Technology \& Digital Equity at AARP. She received her $\mathrm{PhD}$ in communication from the University of Southern California. Dr. Bryant currently heads up AARP's cross-enterprise Technology impact area, which is focused on addressing digital equity for older adults through scalable, long-term solutions. Contact her at abryant@aarp.org.

Lisa Gualtieri, PhD, ScM is Associate Professor at Tufts University School of Medicine in the Department of Public Health and Community Medicine. She received her $\mathrm{PhD}$ in computer science from Harvard University. Dr.
Gualtieri's research lies at the intersection of technology and health, focusing on the design and use of technologies to help people lead healthier lives. Contact her at lisa.gualtieri@tufts.edu. 